International Mathematical Forum, Vol. 8, 2013, no. 11, 513 - 516 HIKARI Ltd, www.m-hikari.com

\title{
Exact Formulae for the Perfect Power Counting Function and the $n$-th Perfect Power
}

\author{
Rafael Jakimczuk \\ División Matemática, Universidad Nacional de Luján \\ Buenos Aires, Argentina \\ jakimczu@mail.unlu.edu.ar
}

Copyright (C) 2013 Rafael Jakimczuk. This is an open access article distributed under the Creative Commons Attribution License, which permits unrestricted use, distribution, and reproduction in any medium, provided the original work is properly cited.

\begin{abstract}
There exist in the literature various exact formulae for the prime counting function $\pi(x)$ and the $n$-th prime $p_{n}$. These formulae use the floor function. In this note we present exact formulae for the perfect power counting function $N(x)$ and the $n$-th perfect power $P_{n}$. These formulae also use the floor function.
\end{abstract}

Mathematics Subject Classification: 11A99, 11B99

Keywords: Perfect powers, exact formulae, counting function, $n$-th perfect power

\section{Introduction}

A natural number of the form $m^{n}$ where $m$ is a positive integer and $n \geq 2$ is called a perfect power. The first few terms of the integer sequence of perfect powers are

$$
1,4,8,9,16,25,27,32,36,49,64,81,100,121,125,128 \ldots
$$

and they form sequence A001597 in Sloane's Encyclopedia.

Let $N(x)$ be the number of perfect powers that do not exceed $x$. In [1] are obtained asymptotic formulae for $N(x)$. For example,

$$
N(x)=\sqrt{x}+\sqrt[3]{x}+\sqrt[5]{x}-\sqrt[6]{x}+\sqrt[7]{x}-\sqrt[10]{x}+\sqrt[11]{x}+o(\sqrt[11]{x})
$$


In this note we obtain an exact formula for $N(x)$ using the floor function.

Let $P_{n}$ be the $n$-th perfect power. That is, $P_{1}=1, P_{2}=4, P_{3}=8, \ldots$. In [2] are obtained asymptotic formulae for $P_{n}$. For example,

$$
P_{n}=n^{2}-2 n^{\frac{5}{3}}-2 n^{\frac{7}{5}}+\frac{13}{3} n^{\frac{4}{3}}-2 n^{\frac{9}{7}}+2 n^{\frac{6}{5}}-2 n^{\frac{13}{11}}+o\left(n^{\frac{13}{11}}\right) .
$$

In this note we obtain exact formulae for $P_{n}$ using the floor function.

In this note (as usual) $\lfloor$.$\rfloor denotes the floor function.$

\section{An exact formula for $N(k)$}

Lemma 2.1 The number $h \geq 2$ is a perfect power if and only if at least one of the numbers

$$
h^{1 / 2}, h^{1 / 3}, \ldots, h^{1 / h}
$$

is a positive integer.

Proof. If $h^{1 / j}=a$, where $a$ is a positive integer and $j \in\{2, \ldots, h\}$, then $h=a^{j}$ is a perfect power.

On the other hand, we have

$$
\begin{gathered}
h<2^{h}<3^{h}<4^{h}<5^{h}<\cdots \\
h<2^{h+1}<3^{h+1}<4^{h+1}<5^{h+1}<\cdots \\
h<2^{h+2}<3^{h+2}<4^{h+2}<5^{h+2}<\cdots
\end{gathered}
$$

Since if $h \geq 3$ then $h<2^{h}$. Consequently if $h \geq 3$ is a perfect power then

$$
h=b^{c},
$$

where $b$ is a positive integer and $c \in\{2, \ldots, h-1\}$. Therefore

$$
h^{1 / c}=b .
$$

The lemma is proved.

Theorem 2.2 We have the following formula

$$
N(k)=1+\sum_{h=2}^{k}\left(1-\prod_{j=2}^{h}\left(1-\left\lfloor\frac{\left\lfloor h^{1 / j}\right\rfloor}{h^{1 / j}}\right\rfloor\right)\right) \quad(k \geq 1)
$$


Proof. Let us consider the function

$$
1-\left\lfloor\frac{\lfloor x\rfloor}{x}\right\rfloor \text {. }
$$

If $x>0$ is an integer then

$$
1-\left\lfloor\frac{\lfloor x\rfloor}{x}\right\rfloor=0 .
$$

On the other hand, If $x>0$ is not an integer then

$$
1-\left\lfloor\frac{\lfloor x\rfloor}{x}\right\rfloor=1 .
$$

Therefore (Lemma 2.1) if $h$ is a perfect power then

$$
1-\prod_{j=2}^{h}\left(1-\left\lfloor\frac{\left\lfloor h^{1 / j}\right\rfloor}{h^{1 / j}}\right\rfloor\right)=1 .
$$

On the other hand (Lemma 2.1) if $h$ is not a perfect power then

$$
1-\prod_{j=2}^{h}\left(1-\left\lfloor\frac{\left\lfloor h^{1 / j}\right\rfloor}{h^{1 / j}}\right\rfloor\right)=0 .
$$

Consequently, formula (1) is proved.

\section{Formulae for $P_{n}$ in terms of $N(k)$.}

We need the following lemma.

Lemma 3.1 We have the following inequality

$$
P_{n} \leq n^{2} \quad(n \geq 1) .
$$

Proof. We have $P_{1}=1^{2}, P_{2}=2^{2}, P_{3}=8<3^{2}$. Consequently if $n \geq 3$ then $P_{n}<n^{2}$. Since the squares are perfect powers. The lemma is proved.

We have the following general theorem.

Theorem 3.2 Suppose that the function $f(x)$ has the following properties.

(i) $f(x)$ is continuous, nonnegative and strictly increasing in the interval $[0, \infty]$

(ii) $f(1)=1$

(iii) $\lim _{x \rightarrow \infty} f(x)=A \leq 2$

Then the following formula holds,

$$
P_{n}=1+\sum_{k=1}^{n^{2}}\left\lfloor f\left(\frac{n}{1+N(k)}\right)\right\rfloor \quad(n \geq 1) .
$$


Proof. Note that in the interval $(0,1)$ we have $0<f(x)<1$ and in the interval $[1, \infty)$ we have $1 \leq f(x)<2$. Besides, we have (Lemma 3.1) $P_{n} \leq n^{2}(n \geq 1)$. Clearly formula (2) is true if $n=1$. Since $N(1)=1$. Suppose that $n \geq 2$.

If $k=P_{n}, P_{n}+1, P_{n}+2, \ldots, n^{2}$ we have (since $N\left(P_{n}\right)=n$ )

$$
0<\frac{n}{1+N(k)}<1
$$

Therefore

$$
\left\lfloor f\left(\frac{n}{1+N(k)}\right)\right\rfloor=0 .
$$

On the other hand, if $k=1,2, \ldots, P_{n}-1$ then $N(k)$ take the values $1,2, \ldots, n-$ 1 and therefore

$$
1 \leq \frac{n}{1+N(k)}
$$

Consequently

$$
\left\lfloor f\left(\frac{n}{1+N(k)}\right)\right\rfloor=1
$$

The theorem is proved.

Example 3.3 A simple family of functions that satisfy conditions (i), (ii) and (iii) is

$$
f(x)=\frac{k x}{(k-1) x+1} \quad(k \geq 2) .
$$

For example, if $k=2$ equation (2) becomes

$$
P_{n}=1+\sum_{k=1}^{n^{2}}\left\lfloor\frac{2\left(\frac{n}{1+N(k)}\right)}{\left(\frac{n}{1+N(k)}\right)+1}\right\rfloor=1+\sum_{k=1}^{n^{2}}\left\lfloor\frac{2 n}{n+1+N(k)}\right\rfloor \quad(n \geq 1) .
$$

Substituting equation (1) into (3) we obtain a formula for $P_{n}$.

ACKNOWLEDGEMENTS. The author is very grateful to Universidad Nacional de Luján.

\section{References}

[1] R. Jakimczuk, On the distribution of perfect powers, J. Integer Seq. 14 (2011), Article 11.8.5.

[2] R. Jakimczuk, Asymptotic formulae for the $n$-th perfect power, J. Integer Seq. 15 (2012), Article 12.5.5.

Received: November, 2012 